\title{
Estudio histórico y epistemológico de la óptica como base para la enseñanza en $2^{\circ}$ de Bachillerato.
}

\author{
Elena Álvarez Jubete ${ }^{a}$, Isabel Hevia Artime ${ }^{b}$, Luigi Toffolatti Ballarin ${ }^{c}$ \\ aProfesora de Educación Secundaria y Bachillerato, eluniversoelegante@live.com, ${ }^{b}$ Profesora del \\ Departamento de Ciencias de la Educación. Universidad de Oviedo heviaisabel@uniovi.les, ${ }^{\mathrm{c}}$ Profesor \\ del Departamento de Física. Universidad de Oviedo ltoffolatti@uniovi.es
}

\begin{abstract}
Resumen
En este trabajo analizamos los textos originales de los grandes científicos sobre la naturaleza de la luz destacando los avances principales que aportaron, y proponemos una relación entre dichas ideas clave y los estándares de aprendizaje que han de ser adquiridas por nuestros alumnos. A partir de estas ideas clave y teniendo en cuenta los posibles obstáculos asociados, desarrollaremos el estudio de la óptica de manera constructiva, para que tanto los alumnos, como el profesor, aprovechen en mayor medida el proceso de enseñanza-aprendizaje.
\end{abstract}

Palabras Clave: óptica, estudio histórico, obstáculo conceptual.

\section{Introducción}

Hay muchas razones para incorporar la historia de la ciencia en la enseñanza de la física (Galili y Hazan, 2016), incluso puede inspirar estrategias de enseñanza que se pueden utilizar como principio organizador del currículo (Matthews, 1994). En nuestro caso, nos permite establecer las ideas clave que tienen que comprender nuestros alumnos, y nos ayuda a detectar los obstáculos que previsiblemente encontrarán los estudiantes, estableciendo un paralelismo entre sus ideas intuitivas y las mantenidas en algún momento de la historia de la ciencia. El desarrollo histórico de las ideas sobre óptica, y las similitudes entre las ideas de las estudiantes y aquellas de los primeros científicos han sido discutidas previamente (Dedes 2005; Galili 1996; Galili y Hazan 2000).

La hipótesis fundamental de la epistemología genética es que hay un paralelismo entre el progreso realizado en la organización lógica y racional del conocimiento (historia de las ciencia), y el proceso psicológico formativo correspondiente (Piaget, 1975). Es por ello que las normas que el sujeto epistémico elabora en el curso de su génesis, podrían ser 
comparables a las normas que son inherentes al pensamiento científico (Piaget y García, 1989). La explicación de cualquier fenómeno ha de buscarse en su propia génesis, lo que confiere un papel muy importante a la historia de la ciencia, tanto en la práctica educativa, como en reflexión sobre la educación.

En este sentido, desde nuestra perspectiva constructivista del aprendizaje, creemos que para comprender bien un concepto hemos de analizarlo desde su origen. Creemos que es muy importante una adecuada adquisición de los conceptos de haz de luz, y rayo de luz para poder comprender la formación de imágenes en la óptica geométrica. Para poder comprender la visión del color consideramos necesario estudiar la naturaleza heterogénea de la luz como solución a las teorías de la modificación. Así mismo, consideramos necesario que los alumnos conozcan que la retina del ojo posee estructuras sensibles a la luz roja, verde y violeta respectivamente, para explicar la visión del color, y que los cambios de curvatura del cristalino tienen como función la acomodación del ojo para la visión de objetos situados a diferentes distancias.

Consideramos el principio de Huygens-Fresnel como base de la explicación de la óptica ondulatoria, así como la necesaria asunción de que la luz se propaga en línea recta y en todas las direcciones en un medio homogéneo, pudiendo producir sombras nítidas, dado que las longitudes de onda de las vibraciones de luz son muy pequeñas comparadas con el tamaño de los objetos visibles. Así mismo consideramos que los alumnos han de comprender que el índice de refracción se explica como la relación entre las velocidades de propagación de las ondas antes y después de la refracción.Por otra parte, han de conocer que la velocidad de la luz que sale de las estrellas es siempre la misma, independientemente de la estrella que la emita, es decir la velocidad de la luz es una constante en nuestro universo.Finalmente creemos que es básico que comprendan que la luz visible es un caso particular de onda electromagnética, es decir es la propagación en el espacio de campos eléctricos y magnéticos perpendiculares.

\section{Presentación de resultados}

En un estudio previo (Hevia-Artime y Álvarez, 2017), habíamos relacionado dichas ideas con las posibles dificultades que los alumnos pueden encontrar para alcanzar los indicadores de comprensión previstos por dicha ley. A continuación, partiendo de las obras originales (que detallamos) presentamos las ideas clave que han permitido avanzar en el estudio de la óptica física, relacionadas con los criterios de calificación que establece la LOMCE (Ley Órgánica par la Mejora de la Calidad Educativa). 
Tabla 1. Relación entre las ideas claves en la enseñanza de la óptica derivadas del estudio de la obra de los principales científicos y criterios de calificación que establece la LOMCE para la asignatura de Física de $2^{\circ}$ de Bachillerato.

\begin{tabular}{|c|c|}
\hline $\begin{array}{l}\text { Ideas claves en la enseñanza de la óptica } \\
\text { derivadas el estudio de la obra de: }\end{array}$ & $\begin{array}{l}\text { Criterios de calificación que establece la LOMCE para } \\
\text { la asignatura de Física de } 2^{\circ} \text { de Bachillerato. }\end{array}$ \\
\hline \multicolumn{2}{|c|}{ Kepler (Kepler, 1604) } \\
\hline $\begin{array}{l}\text { Concepto de rayo de luz } \\
\text { Concepto de haz de luz }\end{array}$ & $\begin{array}{l}\text { Bloque 5. Óptica Geométrica } \\
\text { - "Describir los fenómenos luminosos aplicando el } \\
\text { concepto de rayo". } \\
\text { - "Explicar en qué consiste la aproximación paraxial". } \\
\text { - "Plantear gráficamente la formación de imágenes en el } \\
\text { dioptrio plano y en el dioptrio esférico". } \\
\text { - "Explicar la formación de imágenes en espejos y lentes } \\
\text { delgadas trazando correctamente el esquema de rayos } \\
\text { correspondiente e indicando las características de las } \\
\text { imágenes obtenidas". } \\
\text { - "Explicar el funcionamiento de algunos instrumentos } \\
\text { ópticos (lupa, microscopio, telescopio y cámara } \\
\text { fotográfica) utilizando sistemáticamente los diagramas de } \\
\text { rayos para obtener gráficamente las imágenes". }\end{array}$ \\
\hline \multicolumn{2}{|c|}{ Newton (Newton, 1704) } \\
\hline $\begin{array}{l}\text { La luz "blanca" es de naturaleza } \\
\text { heterogénea. } \\
\text { La luz no se modifica al interaccionar con } \\
\text { los objetos. }\end{array}$ & $\begin{array}{l}\text { Bloque 4. Ondas } \\
\text { - "Explicar por qué y cómo se perciben los colores de los } \\
\text { objetos". }\end{array}$ \\
\hline \multicolumn{2}{|c|}{ Grimaldi (Grimaldi, 1665) } \\
\hline $\begin{array}{l}\text { La luz se propaga no sólo de manera } \\
\text { directa, por refracción o por reflexión, sino } \\
\text { que existe un cuarto modo, por difracción }\end{array}$ & $\begin{array}{l}\text { Bloque 4. Ondas } \\
\text { 7. "Reconocer la difracción y las interferencias como } \\
\text { fenómenos propios del movimiento ondulatorio". }\end{array}$ \\
\hline \multicolumn{2}{|c|}{ Hooke (Hooke, 1665) } \\
\hline $\begin{array}{l}\text { La luz se propaga en línea recta y en todas } \\
\text { las direcciones en un medio homogéneo. } \\
\text { La velocidad de la luz, aunque muy } \\
\text { grande, es finita. La luz ha de ser un } \\
\text { movimiento de vibración muy rápido y } \\
\text { esta vibración ha de ser muy corta. }\end{array}$ & $\begin{array}{l}\text { Bloque 5. Óptica geométrica } \\
\text { - "Describir los fenómenos luminosos aplicando el } \\
\text { concepto de rayo". } \\
\text { Germen para el desarrollo de la teoría ondulatoria de } \\
\text { Huygens. }\end{array}$ \\
\hline
\end{tabular}




\begin{tabular}{|c|c|}
\hline \multicolumn{2}{|c|}{ Huygens (Huygens, 1690) } \\
\hline $\begin{array}{l}\text { La luz es un movimiento sucesivo impreso } \\
\text { a la materia. } \\
\text { La luz se propaga mediante ondas } \\
\text { esféricas. } \\
\text { Todos los puntos de un frente de ondas son } \\
\text { centros emisores de ondas secundarias. } \\
\text { De todo centro emisor se propagan ondas } \\
\text { en todas las direcciones del espacio con } \\
\text { velocidad distinta en cada medio. } \\
\text { Las ondas se cruzan sin confusión y sin } \\
\text { destruirse unas a otras. } \\
\text { Cada punto del espacio al que le llega una } \\
\text { onda, se comporta él mismo como emisor } \\
\text { de una onda esférica. }\end{array}$ & $\begin{array}{l}\text { Bloque 4. Ondas } \\
\text { - "Visualizar gráficamente la propagación de las ondas } \\
\text { mediante frentes de onda y explicar el fenómeno } \\
\text { empleando el principio de Huygens". } \\
\text { - "Reconocer la difracción y las interferencias como } \\
\text { fenómenos característicos de las ondas y que las partículas } \\
\text { no experimentan". } \\
\text { - "Explicar los fenómenos de interferencia y la difracción } \\
\text { a partir del Principio de Huygens". }\end{array}$ \\
\hline $\begin{array}{l}\text { El índice de refracción se explica como la } \\
\text { relación entre las velocidades de } \\
\text { propagación de las ondas antes y después } \\
\text { de la refracción. }\end{array}$ & $\begin{array}{l}\text { Bloque 4. Ondas } \\
\text { - "Definir el concepto de índice de refracción e interpretar } \\
\text { la refracción como una consecuencia de la modificación } \\
\text { en la velocidad de propagación de la luz al cambiar de } \\
\text { medio". }\end{array}$ \\
\hline \multicolumn{2}{|c|}{ Malebranche (Ferraz, 1974) } \\
\hline $\begin{array}{l}\text { Explica por primera por primera vez la } \\
\text { relación entre los colores y las distintas } \\
\text { velocidades de vibración }\end{array}$ & $\begin{array}{l}\text { Bloque 4. Ondas } \\
\text { - "Reconocer la dependencia del índice de refracción de } \\
\text { un medio con la frecuencia y justificar el fenómeno de la } \\
\text { dispersión". }\end{array}$ \\
\hline \multicolumn{2}{|c|}{ Euler (Euler, 1746) } \\
\hline $\begin{array}{l}\text { Introduce la noción de frecuencia de la } \\
\text { vibración luminosa para explicar los } \\
\text { colores. } \\
\text { Atribuye la frecuencia más alta de la } \\
\text { vibración luminosa al color violeta, y la } \\
\text { más baja al color rojo }\end{array}$ & $\begin{array}{l}\text { Bloque 4. Ondas } \\
\text { - "Relacionar la visión de colores con la frecuencia". } \\
\text { - "Explicar por qué y cómo se perciben los colores de los } \\
\text { objetos". }\end{array}$ \\
\hline $\begin{array}{l}\text { Considera la luz como una onda elástica } \\
\text { sinusoidal que se propaga en el éter. } \\
\text { Unifica los fenómenos eléctricos y } \\
\text { magnéticos, proponiendo el éter luminoso. }\end{array}$ & Germen de la teoría de Young y posteriormente Maxwell \\
\hline
\end{tabular}


Bradley (Bradley, 1728)

\begin{tabular}{|c|c|}
\hline \multicolumn{2}{|c|}{ Bradley (Bradley, 1728) } \\
\hline $\begin{array}{l}\text { La velocidad de la luz que sale de las } \\
\text { estrellas es siempre la misma, } \\
\text { independientemente de la estrella que la } \\
\text { emita. }\end{array}$ & $\begin{array}{l}\text { Bloque 4. Ondas } \\
\text { - "Conocer el debate histórico sobre la naturaleza de la luz } \\
\text { y el triunfo del modelo ondulatorio e indicar razones a } \\
\text { favor y en contra del modelo corpuscular". }\end{array}$ \\
\hline \multicolumn{2}{|c|}{ Malus (Malus, 1810) } \\
\hline $\begin{array}{l}\text { La doble refracción no era el único modo } \\
\text { de polarizar la luz. La luz reflejada } \\
\text { también podía tener "lados". }\end{array}$ & $\begin{array}{l}\text { Bloque 4. Ondas } \\
\text { 15. "Comprender las características y propiedades de } \\
\text { las ondas electromagnéticas, como su longitud de onda, } \\
\text { polarización o energía, en fenómenos de la vida } \\
\text { cotidiana". }\end{array}$ \\
\hline \multicolumn{2}{|c|}{ Young (Young, 1802) } \\
\hline $\begin{array}{l}\text { La retina del ojo posee estructuras } \\
\text { sensibles a la luz roja, verde y violeta } \\
\text { respectivamente. }\end{array}$ & $\begin{array}{l}\text { Bloque 4. Ondas } \\
\text { - "Relacionar la visión de colores con la frecuencia". } \\
\text { - "Explicar por qué y cómo se perciben los colores de los } \\
\text { objetos". }\end{array}$ \\
\hline $\begin{array}{l}\text { Los cambios de curvatura del cristalino } \\
\text { tienen como función la acomodación del } \\
\text { ojo para la visión de objetos situados a } \\
\text { diferentes distancias. }\end{array}$ & $\begin{array}{l}\text { Bloque 5. Óptica Geométrica } \\
\text { - "Describir el funcionamiento óptico del ojo humano". }\end{array}$ \\
\hline $\begin{array}{l}\text { Dos ondas de luz que se superponen } \\
\text { pueden interferir la una con la otra. }\end{array}$ & $\begin{array}{l}\text { Bloque 4. Ondas } \\
\text { - "Conocer el debate histórico sobre la naturaleza de la luz } \\
\text { y el triunfo del modelo ondulatorio e indicar razones a } \\
\text { favor y en contra del modelo corpuscular". }\end{array}$ \\
\hline $\begin{array}{l}\text { La luz viaja en línea recta, pudiendo } \\
\text { producir sombras nítidas, dado que las } \\
\text { longitudes de onda de las vibraciones de } \\
\text { luz son muy pequeñas comparadas con el } \\
\text { tamaño de los objetos visibles. }\end{array}$ & $\begin{array}{l}\text { - "Explicar fenómenos cotidianos (los espejismos, el arco } \\
\text { iris, el color azul del cielo, los patrones en forma de } \\
\text { estrella que se obtienen en algunas fotografías de fuentes } \\
\text { de luz, entre otros) como efectos de la reflexión, } \\
\text { difracción e interferencia". } \\
\text { - "Reconocer la difracción y las interferencias como } \\
\text { fenómenos característicos de las ondas y que las partículas } \\
\text { no experimentan". }\end{array}$ \\
\hline \multicolumn{2}{|r|}{ Young - Fresnell } \\
\hline $\begin{array}{l}\text { Las ondas de luz son periódicas y el color } \\
\text { es función de la longitud de onda. } \\
\text { La luz es una onda de naturaleza } \\
\text { transversal }\end{array}$ & Germen de la teoría de Maxwell \\
\hline
\end{tabular}




\begin{tabular}{|c|c|}
\hline \multicolumn{2}{|c|}{ Fresnell (Fresnell, 1827) } \\
\hline Principio de Huygens-Fresnell & $\begin{array}{l}\text { Bloque 5. Óptica Geométrica } \\
\text { - "Explicar en qué consiste la aproximación paraxial". }\end{array}$ \\
\hline Explicación de la difracción & $\begin{array}{l}\text { Bloque 4. Ondas } \\
\text { - "Reconocer la difracción y las interferencias como } \\
\text { fenómenos característicos de las ondas y que las partículas } \\
\text { no experimentan". } \\
\text { - "Explicar los fenómenos de interferencia y la difracción } \\
\text { a partir del Principio de Huygens". }\end{array}$ \\
\hline Explicación de la dispersión de la luz & $\begin{array}{l}\text { Bloque 4. Ondas } \\
\text { - "Reconocer la dependencia del índice de refracción de } \\
\text { un medio con la frecuencia y justificar el fenómeno de la } \\
\text { dispersión". }\end{array}$ \\
\hline \multicolumn{2}{|c|}{ Maxwell (Maxwell, 1865) } \\
\hline $\begin{array}{l}\text { La luz visible es un caso particular de onda } \\
\text { electromagnética. } \\
\text { Explica matemáticamente las ondas } \\
\text { electromagnéticas como la propagación de } \\
\text { campos eléctricos y magnéticos } \\
\text { perpendiculares }\end{array}$ & $\begin{array}{l}\text { Bloque 4. Ondas } \\
\text { - "Identificar las ondas electromagnéticas como la } \\
\text { propagación de campos eléctricos y magnéticos } \\
\text { perpendiculares". } \\
\text { - "Reconocer las características de una onda } \\
\text { electromagnética polarizada y explicar gráficamente el } \\
\text { mecanismo de actuación de los materiales polarizadores". } \\
\text { - "Relacionar la velocidad de la luz con las constantes } \\
\text { eléctrica y magnética". } \\
\text { - "Identificar las ondas electromagnéticas que nos rodean } \\
\text { y valorar sus efectos en función de su longitud de onda y } \\
\text { energía". } \\
\text { - "Describir el espectro electromagnético, ordenando los } \\
\text { rangos en función de la frecuencia, particularmente el } \\
\text { infrarrojo, el espectro visible y el ultravioleta, } \\
\text { identificando la longitud de onda asociada al rango visible } \\
\text { (alrededor de } 500 \text { nm)". } \\
\text { - "Evaluar la relación entre la energía transferida por una } \\
\text { onda y su situación en el espectro electromagnético". }\end{array}$ \\
\hline
\end{tabular}




\begin{tabular}{|c|c|}
\hline & Hertz \\
\hline $\begin{array}{l}\text { Producir por primera vez en el laboratorio } \\
\text { radiación electromagnética } \\
\text { Descubrir un detector capaz de detectar } \\
\text { dichas ondas. } \\
\text { Las ondas electromagnéticas se reflejaban, } \\
\text { se refractaban y podían difractarse y } \\
\text { polarizarse como las ondas de luz, } \\
\text { viajando en línea recta con una velocidad } \\
\text { que era del mismo orden que la velocidad } \\
\text { de la luz. }\end{array}$ & $\begin{array}{l}\text { - "Explicar cómo se generan las ondas de la } \\
\text { radiofrecuencia". } \\
\text { - "Reconocer la importancia de las ondas } \\
\text { electromagnéticas en las telecomunicaciones (radio, } \\
\text { telefonía móvil, etc.)". } \\
\text { - "Identificar distintos soportes o medios de transmisión } \\
\text { (los sistemas de comunicación inalámbricos o la fibra } \\
\text { óptica y los cables coaxiales, entre otros) y explicar de } \\
\text { forma esquemática su funcionamiento". }\end{array}$ \\
\hline
\end{tabular}

\section{Análisis y discusión de resultados.}

Nuestro análisis histórico de las obras de los principaeles autores sobre la naturaleza de la luz, en busca de un paralelismo entre el desarrollo de las diferentes teorías y la construcción de los conceptos que realizan los estudiantes durante el aprendizaje de la óptica geométrica $\mathrm{y}$ ondulatoria, nos ha permitido encontrar las ideas clave que hemos de lograr que comprendan nuestros alumnos. En este trabajo hemos mostrado su relación con los criterios de evaluación planteados en el marco de la actual ley educativa, en base a los cuales los alumnos serán evaluados. Hemos comprobado como efectivamente, las ideas que nosotros consideramos clave en el desarrollo de la óptica, y que mostramos destacadas para cada autor en el cuadro anterior, son la base de los criterios de calificación, y por tanto han de ser adquiridas por nuestros alumnos, y en base a ellas ha desarrollarse la práctica educativa.

\section{Conclusiones}

A partir de estas ideas clave en la enseñanza de la óptica, teniendo en cuenta los posibles obstáculos asociados, se puede desarrollar el estudio de la óptica de manera constructiva, para que tanto los alumnos, como el profesor, aprovechen en mayor medida el proceso de enseñanza-aprendizaje. Hemos de basar nuestra enseñanza en la adquisición de dichas ideas por parte de los alumnos. 


\section{Referencias}

Hevia-Artime, I.\& Álvarez, E. (2017). Trabajo por proyectos: estudio de la Óptica en $2^{\circ}$ de Bachillerato. Actas INNODOCT/17. International Conference on Innovation, Documentation and Education. Valencia: Universitat Politècnica de València (en prensa)

Bradley, J. (1728). IV. A letter from the Reverend Mr. James Bradley Savilian Professor of Astronomy at Oxford, and F. R. S. to Dr. Edmond Halley Astronom. Reg. \&c. giving an account of a new discovered motion of the fixed stars. Philosophical Transactions. 1728, 35, 637-661. doi:10.1098/rstl.1727.0064

Dedes, C. (2005). The Mechanism of Vision: Conceptual Similarities between Historical Models and Children's Representations. Science Education, 14, 699-712.

Euler, L. (1746). "Nova theoria lucis et colorum". Euler Archive - All Works. 88. https://scholarlycommons.pacific.edu/euler-works/88

Ferraz, A. (1974). Teorías sobre la naturaleza de la luz. De Pitágoras a Newton, Madrid: Dossat.

Fresnel, A. (1827). Théorie mécanique de la doublé réfraction. Memoires de L’Académie Royal des Sciences. Paris, Francia: Firmin Didot, Père et files Libraires.

Galili, I. (1996). Student's conceptual change in geometrical optics. International Journal of Science Education, 18 (7), 847-868.

Galili, I. (2016). From Comparison Between Scientists to Gaining Cultural Scientific Knowledge: Leonardo and Galileo. Science and Education, 25, 115-145.

Galili, I., \& Hazan, A. (2000). Learners' knowledge in optics: interpretation, structure and analysis. International Journal of Science Education, 22, 1, 57-88.

Grimaldi, F. M. (1665). Physico-mathesis de lumine, coloribus, et iride. (Edición 2017). Delhi: Relnk Books.

Hooke, R. (1665). Micrographia, or some physiological descriptions of minute bodies made by magnifying glasses? With observations and inquiries thereupon. Openlibrary edition OL6528209M.

Huygens, C. (1690). Traité de la Lumière. Pierre Vander: Leiden; Dunod, Paris, 1992.

Kepler, J. (1604). Les fondaments de l'optique moderne. Paralipomènes a Vitellion (Edición 1980). París, Francia: Vrin.

Malus, E.L. (1810). Memóire, Compte rendus des sciences de l'Académie des Sciences, Tome 33.

Maxwell, J. C. (1865). A Dynamical Theory of the Electromagnetic Field. Eastford: Martino Fine Books. (2013 Reprint)

Matthews, M. R. (1994). Historia, filosofía y enseñanza de las ciencias: la aproximación actual. Enseñanza de las Ciencias, 12 (2), 255-277. 
Maxwell, J. C. (1865). A Dynamical Theory of the Electromagnetic Field. Eastford, EEUU: Martino Fine Books. (2013 Reprint)

Newton, I. (1704). Óptica o tratado de las reflexiones, refracciones, inflexiones y colores de la luz (Edición 1979). Madrid: Alfaguara.

Piaget, J. (1975). Introducción a la epistemología genética. EI pensamiento físico. Buenos Aires: Ed. Paidós.

Piaget J., \& García, R. (1989). Psychogenesis and the history of science. New York, EEUU: Columbia University Press.

Young, T. (1802). The Bakerian Lecture: On the theory of light and colours. London, UK: Philosophical Transactions, Royal Society. 